\title{
Evaluation on Construction of Well-being Cities By Entropy-Weight Based TOPSIS Method
}

\author{
Zhao Yong a,* Chen Songlin ${ }^{\mathrm{b}}$ Lian Ruiruic $^{\mathrm{c}}$ Liu Lili $^{\mathrm{d}}$ \\ Research Center of Urban Management \\ Anhui Jianzhu University, Hefei 230601 China \\ ahfyongzhao@163.com, bsonglin0001@163.com \\ Irr2006@tom.com, diuliil72868@163.com
}

Keywords: Well-being city; Entropy weight method; TOPSIS method; Middle region

\begin{abstract}
Construction of well-being city has already become the development objective of more and more cities. This paper established the evaluation indicator system for construction of well-being city from two dimensions, i.e. public service and city development based on definition of connotation of a well-being city, built the evaluation model for construction of a well-being city by entropy-weight based TOPSIS method and evaluated the construction situation of well-being city in the six provincial capital cities in Middle China.

With the development of the society and the continuous improvement of living standard, people not only pursue the constant improvement of their physical life level, but also hope to have a happy life. Based on the strong concern on the quality of life and sense of well-being, some countries and regions turn the original single economic indexes of government blindly pursuing GDP to more diverse social evaluation standard such as well-being indexes, and put forward the development objective for building well-being cities. Based on predecessors' study on city well-being indicator system, this paper evaluated the construction of well-being city of 6 provincial capital cities in Middle China by the entropy weight based TOPSIS method, analyzed the main influencing factors in construction of well-being city in order to provide theoretical guidance for construction of well-being city in every region.
\end{abstract}

\section{Connotation of Well-being City}

The perception level of residents of well-being in the city they live is an important indicator to measure whether a city is a well-being city or not, and is usually expressed by the well-being index. Because everyone has different view of well-being, and the social class where they stay is different, there is a big difference in their understanding and feeling of well-being. However, the sense of well-being still has some common characteristics, which mainly includes sense of health, sense of well-off, sense of stability, sense of belonging, sense of identity, sense of joy and sense of satisfaction, etc. ${ }^{[1]}$. After all, the sense of well-being is a kind of subjective feeling; it is not only a factual judgment of the objective conditions of life and state, but also a kind of value judgment of the subjective meaning and degree of satisfaction. It manifests as a positive psychological experience produced on the basis of life satisfaction, and is closely related to many factors, such as income, housing, marriage, family, health, insurance, education, employment, etc. and, and changes periodically with the development of economy and society on the basis of this ${ }^{[2,3]}$.

Construction of well-being city fully embodies the concept of human-oriented management in the aspect of city management guiding ideology. The traditional city management is the city management activity with the government as the sole subject, its characteristics not only lie in that the government is the only subject, but also uses power control, administrative commands and system constraints as the main means in terms of management methods and means, therefore, the tendency of inhumane nature of management is prominent. The construction of well-being city focuses on the subjective feeling of urban residents, which reflects the change of urban management from 
government-oriented management to citizens-oriented management, is favorable to promoting the shift of government management mode from constraint and control to service and intimacy to citizens, truly reflects the guiding ideology of governing for the people and paying attention to people's livelihood, promotes the city managers to make sensitive response to the need of citizens, and further promotes the improvement of the government management efficiency ${ }^{[4]}$.

Building a well-being city must take the public well-being as the government's administration objective, and constrains income differentiation and the overdraft of growth from ecological environment and resources to a certain extent. To realize maximized well-being, the ecological environment, natural resources and the government's public service will face a great challenge.

\section{Establishment of Well-being City Evaluation Indicator System}

For the construction of well-being city, scholars generally adopt city well-being index for evaluation and measurement. Scholars at home and abroad conducted study and measurement of well-being index, and developed their own indicator system. Some scholars adopt factors, such as the Gini coefficient, unemployment rate, gross domestic product, social health, social welfare, social civilization, ecological environment etc. as the basis for calculating gross national well-being indexes.

Cummins from Deakin University, Australia divides well-being index (PWI) into personal well-being index (PWI) and national well-being index (NWI).The national well-being indexes consist of evaluation of people on six aspects such as the current economic situation of the country, the condition of natural environment, social condition, the government, business situation and situation of national security ${ }^{[5]}$. Daniel, et al from Princeton University adopt the ex-post recall reconstruction method for measurement ${ }^{[6]}$; the well-being index released by the world value research institution in the charge of Ronald, professor of University of Michigan has only one question: put all the things together, you think you are very happy, relatively happy, not very happy or not happy ${ }^{[7]}$. The International University Survey developed by Edward, psychology professor of University of Illinois, et al, takes the theory of subjective well-being as the basis, including the contents of five aspects such as the general subjective well-being, life satisfaction, positive emotion, negative emotion, external criteria and self-experience ${ }^{[8]}$. Cummins developed the personal well-being schedule, which requires people to evaluate degree of satisfaction in such aspects as of their own living standard, health, achievements in life, interpersonal relationship, security situation, social involvement, their future security ${ }^{[5]}$. The domestic scholar Xing Zhanjun raised that the well-being is composed of enjoying development experience and mental-physical health experience and can be further broken down into 10 secondary evaluation indicators: experience index of contentment and abundance, mental health experience index, growth and development experience index, social confidence experience index, target value experience index, self-acceptance experience index, interpersonal adjustment experience index, physical health experience index, mentality balance experience index and family atmosphere experience index ${ }^{[9]}$. There are some scholars with a background in economics and statistics who proposed the well-being index measurement and evaluation indicators from the viewpoint of macro-policy and management, for example, Zhuang Lianping and Li Jing put forward 11 types of well-being indexes based on the general well-being, including working condition, leisure condition, inequality, social relations, community welfare, psychological well-being, authorization, political freedom, economic stability, political security and environmental conditions ${ }^{[10]}$.

Based on previous studies and according to the connotation of the well-being city and the principles of scientificity, integrity, measurability and operability etc, of indicator establishment, this paper builds the evaluation indicator system for construction of well-being city from two levels, i.e. urban "public service" and "city development" and selects six second-level indicators and 24 third-level indicators in total. This paper takes into account the data availability and gives up part of indicators while selecting the indicators. 


\section{Establishment of Evaluation Model by Entropy Weight Based TOPSIS Method}

TOPSIS (Technique for order preference by similarity to an ideal solution) method, is an ideal technique for order preference by similarity to an ideal solution ${ }^{[11-13]}$. Its basic principle is to prioritize by measuring the vector of indicator evaluation value of the evaluated target and the relative distance between the ideal solution and negative ideal solution of comprehensive evaluation problem. The so-called ideal solution $\left(\mathrm{x}^{+}\right)$is an ideal optimal solution, the value of its each indicator all reaches the optimal value of each indicator in the comprehensive evaluation problem, therefore, it is also known as the optimal solution; the so-called negative ideal solution (marked as $\mathrm{X}^{-}$) is another supposed worst solution, the value its each indicator all reaches the worst value of each indicator in the comprehensive evaluation problem, therefore it is also known as the worst solution. Solution ranking rule is to compare each alternative solution with $\mathrm{x}^{+}$and $\mathrm{x}^{-}$, if one of the solutions is closest to $\mathrm{x}^{+}$and farthest from the $\mathrm{x}^{-}$at the same time, then this solution is the best solution of alternative solutions.

TOPSIS method can be divided into the following steps:

(1) Standardization of indicator data

Positive indicators mean those the better, the bigger whose values are, whereas negative indicators are those the better, the smaller whose values are. Suppose ${ }^{y_{i j}}$ represents the value of $i^{\text {th }}$ evaluation object after standardization processing of $\mathrm{j}^{\text {th }}$ indicator, and $X_{i j}$ represents the indicator value of $i^{\text {th }}$ evaluation object for $\mathrm{j}^{\text {th }}$ indicator, Then, the standardization formula of positive indicator data is as follows:

$y_{i j}=\frac{x_{i j}-x_{j}^{\min }}{x_{j}^{\max }-x_{j}^{\min }}$

And the standardization formula of negative indicator data is as follows:

$y_{i j}=\frac{x_{j}^{\max }-x_{i j}}{x_{j}^{\max }-x_{j}^{\min }}$

where $x_{j}^{\max }=\max \left\{x_{i j}\right\}, x_{j}^{\min }=\min \left\{x_{i j}\right\}$

Process $p_{i j}$ by normalization:

$$
P_{i j}=\frac{y_{i j}}{\sum_{i=1}^{m} y_{i j}}
$$

(2) Determination of weight $w_{j}$ by entropy weight

Calculate the entropy of $\mathrm{j}^{\text {th }}$ indicator:

$$
H_{j}=-\sum_{i=1}^{m} p_{i j} \ln p_{i j}
$$

Calculate difference of indicator:

$$
K_{j}=1-\frac{H_{i j}}{\ln m}
$$

Calculate the entropy weight of each indicator.Set ${ }^{w_{j}}$ to be the entropy weight of $\mathrm{j}^{\text {th }}$ evaluation indicator, then we get:

$$
w_{j}=\frac{k_{j}}{\sum_{j=1}^{n} K_{j}}
$$

It can be seen from above formula that the entropy weight has following character: when that is, when $P_{i 1}=P_{i 2}=\cdots P_{i n}$, the value of entropy ${ }_{j}$ reaches the maximum value, at this time the entropy 
weight is equal to zero, that is to say, the $\mathrm{j}^{\text {th }}$ indicator does not provide any useful information to decision makers, so this indicator can be deleted. The bigger the entropy indicator, the smaller its entropy weight, and the less important this indicator. Considering from viewpoint of information, the entropy weight represents the degree that this indicator provides the amount of useful information in the problem. So, the calculated entropy weight can be used as attribute weight, the larger the entropy weight, the bigger the weight, and the more important the attribute of corresponding indicator.

(3) Method of determining evaluation score by TOPSIS method

Weight the value of standardization indicator:

The weighted normalized decision-making matrix is constituted by normalized decision-making matrix $y=\left(y_{i j}\right)_{m \times n}$ and weight vector $w=\left(w_{1}, w_{2}, w_{3}, \cdots, w_{n}\right)$ :

$A=\left(a_{i j}\right)_{m \times n}=\left(w_{i j}\right)_{m \times n}$

Determine the ideal solution and negative ideal solution to constitute the vector of ideal solution $\mathrm{x}+$ and the vector of negative ideal solution $\mathrm{x}-$, respectively:

$x^{+}=\left\{a_{j}^{+}\right\}(j=1,2,3, \cdots, n) ; x^{-}=\left\{a_{j}^{-}\right\}(j=1,2,3, \cdots, n)$

Where,

$$
\begin{aligned}
& a_{j}^{+}=\max \left(a_{1 j}, a_{2 j}, a_{3 j}, \cdots, a_{m j}\right)(j=1,2,3, \cdots, n) \\
& a_{j}^{-}=\min \left(a_{1 j}, a_{2 j}, a_{3 j}, \cdots, a_{m j}\right)(j=1,2,3, \cdots, n)
\end{aligned}
$$

Calculate the distance $\mathrm{d}^{+}$of the vector of indicator evaluation value of the evaluated object to ideal solution $\mathrm{x}^{+}$and distance $\mathrm{d}^{-}$to negative ideal solution $\mathrm{x}^{+}$:

$$
d_{i}^{+}=\sqrt{\sum_{j=1}^{n}\left(a_{i j}-a_{j}^{+}\right)^{2}}, \quad d_{i}^{-}=\sqrt{\sum_{j=1}^{n}\left(a_{i j}-a_{j}^{-}\right)^{2}} \quad i=1,2,3, \cdots, m
$$

Calculate the distance of vector of indicator evaluation value of each evaluated object to the ideal solution:

$$
c_{j}=100 \times \frac{d_{i}^{-}}{d_{i}^{+}+d_{i}^{-}} \quad i=1,2,3, \cdots, m
$$

\section{Empirical Study on Well-being City Construction Evaluation}

\section{Sample selection and data sources}

This paper selects such six provincial capital cities in Middle China as Hefei, Wuhan, Changsha, Zhengzhou, Taiyuan and Nanchang as the solid evidence to evaluate the construction situation of well-being city in the capital cities of the six provinces in Middle China according to the above evaluation indicator system for construction of well-being city. Raw data of evaluation indicators comes from annual statistical yearbook, annual statistical yearbook on environment protection and environment quality bulletin, etc. of each province. Among all above evaluation indicators, C3, C22 and C34 are negative type indicators, and other indicators are all positive indicators. Substitute the data of positive indicators in raw data of each indicator in Table 1 into Eq.1, and substitute the data of negative indicator into Eq.2, we can get the value of standardized indicator.

\section{Evaluation of Well-being city construction by TOPSIS method based on entropy weight}

(1)Calculation of indicator weight by entropy weight method

Substitute the value of each standardized indicator in Table 2 into Eq.3 5, we can get the

entropy value $K_{j}$ of each evaluation indicator, and substitute the entropy value into Eq.6, we can get entropy weight $W_{j}$ of each indicator, the indicator weights are available, respectively according to the weight of each level.

(2)Calculation of comprehensive evaluation and score of each level by TOPSIS 
Substitute the value of standardized indicator and weight of indicator into Eq.8 10, then we can get the vector of ideal solution and vector of negative ideal solution:

$x^{+}=$Where, $x^{+}$represents the value of each indicator reaches the optimal value of each indicator in comprehensive evaluation of well-being, so, it is also known as the optimal solution; $X^{-}$represents the value of each indicator reaches the worst value of each indicator in the comprehensive evaluation of well-being, so it is also known as the worst solution.

Substitute the matrix A of weighted standardized indicator and ideal solution $\mathrm{d}^{+}$and $\mathrm{d}^{-}$into Eq.11 12, then we can get the comprehensive evaluation score of each sample, as shown in line 9 "composite" in Table 1. Substitute the value of weighted standardized indicator into Eq.11 12, then we can get the score of each sample at each level, as listed in lines 1 8 in Table 1.

Tab.1 Composite scores of well-being cities in the six provincial capital cities in Middle China and score result of indicator at each level

\begin{tabular}{|c|c|c|c|c|c|c|}
\hline & HF & CS & WH & ZZ & TY & NC \\
\hline C1 & 30.70 & 46.02 & 69.70 & 53.00 & 33.33 & 28.20 \\
\hline C2 & 42.17 & 68.00 & 89.02 & 38.73 & 53.74 & 61.27 \\
\hline C3 & 49.25 & 63.62 & 35.88 & 53.59 & 41.49 & 46.03 \\
\hline C4 & 35.49 & 26.57 & 56.66 & 59.63 & 44.38 & 43.94 \\
\hline C5 & 51.41 & 51.08 & 52.53 & 77.89 & 38.23 & 16.30 \\
\hline C6 & 41.67 & 23.90 & 61.16 & 33.84 & 41.62 & 33.58 \\
\hline B1 & 43.46 & 54.76 & 67.02 & 44.98 & 26.92 & 21.19 \\
\hline B2 & 47.23 & 43.88 & 53.09 & 38.57 & 32.76 & 63.20 \\
\hline Composite & 43.32 & 42.15 & 45.39 & 41.77 & 38.94 & 40.80 \\
\hline
\end{tabular}

\section{Analysis of evaluation results}

Firstly, from the perspective of the weights determined by entropy weight method, six second-level indicators have different degree of influence on the construction of well-being cities. Where C1 (27.8\%) and C2 (27.9\%) have biggest influence, next are C5 (13.2\%) and C4(13.1\%), C3 $(9.9 \%)$ and C6 (8.1\%) have relatively small influence. Two first-level indicators have different degree of influence on evaluation of well-being city construction, among them the city development B1 $(65.6 \%)>$ public service B2 $(34.4 \%)$.

Secondly, from the perspective of comprehensive evaluation scores, the order of evaluation scores for construction of well-being cities of the six provincial capital cities in Middle China from big to small is Wuhan $>$ Hefei $>$ Changsha $>$ Zhengzhou $>$ Nanchang $>$ Taiyuan.

Thirdly, from comprehensive evaluation scores of well-being cities of the six provincial capital cities in Middle China and evaluation score of indicator at each level, the construction of well-being city of each city has its own features:

The order of scores for "city development" in first-level indicators from big to small is Wuhan $>$ Changsha $>$ Zhengzhou $>$ Hefei $>$ Taiyuan $>$ Nanchang. It can be seen from second-level indicators in Table 4 that the economic development indicators of Hefei city are the main factors that restrict the well-being city construction of Hefei city; and it can be seen from third-level indicators in Table 2 that the scores for per capita GDP and per capita disposable income are relatively low, which is the main factor that Hefei city gets lower scores for its construction of well-being city.

The order of scores for "public service" in first-level indicators from big to small is Nanchang $>$ Wuhan $>$ Hefei $>$ Changsha $>$ Zhengzhou $>$ Taiyuan. Where, it can be seen from second-level indicators in Table 4 that the living environment indicators of Wuhan city are the main factors that restrict the construction of well-being city of Wuhan city; and it can be seen from third-level indicators in Table 2 that the per capita park green land area and excellent air quality rate get relatively low scores, which is the main factor that the score of Wuhan city for its construction of well-being city is relatively low. 


\section{Conclusions}

Construction of well-being city needs to have a set of scientific indicator system for follow-up, evaluation, guidance and practice. Based on definition of the connotation of well-being city, this paper established the indicator system with six second-level indicators and 24 third-level indicators from two dimensions of public service and urban development; established evaluation model for the construction of well-being city by entropy weight based TOPSIS method, and analyzed and evaluated advantages and disadvantages of the six provincial capital cities of Middle China in the construction of well-being and the main influencing factors. The research results show that using the indicator system for evaluation of well-being city and evaluation method devised in this paper can effectively monitor and measure the construction progress of well-being city and search for ways to enhance or improve degree of city well-being.

\section{Acknowledgements}

This work was supported by Humanities and Social Sciences Project of Anhui Education Department(SK2015A09 、 SK2015A298) ; Anhui Philosophy Social Sciences Plan Project(AHSKY2014D16); Humanities and Social Sciences Project of Anhui Education Department(SK2014A204).

\section{References}

[1] Andrew J. Happiness and economic performance. The Economic Journal, 1997(107):445-446.

[2] Hilke Brockmann etc. The China puzzle: Falling happiness in a rising economy. Journal of Happiness Study, 2009(10):721-732.

[3] Li Cong-ming. New frame for measurement of national wealth: GNH+GDP pattern [J]. Transaction of Xi'an Financial Services Institute, 2005(3):34-38.

[4] Chen Zhi-xia. City happiness indexes and their measurement and evaluation index system. City Issue, 2012(4):9-13.

[5] Robert A Cummins. Objective and subjective quality of life: An interactive model. Social Indicators Research. 2000(52):55-72.

[6] Daniel Kahneman, Alan B Krueger. Developments in the measurement of subjective well-being. The Journal of Economic Perspectives. 2006(20):3-24.

[7] Welzel C, Ronald F Inglehart. Agency, values, and well-being: A human development model. Social Indicators Research. 2010(1):43-63.

[8] Edward. Subjective well-being: The science of happiness and proposal for national index. American psychologist, 2000(1):34-43.

[9] Xing Zhan-jun. Establishment and follow-up study of index system for happiness index. Data, 2006(8):10-12.

[10]Zhuang Lian-ping, Li Jing. Establishment of general gross national happiness index based on correlation analysis. Statistics Education, 2008(8):36-40.

[11]Luo Dang, Wang Shu-ying. Decision-making theory and method. Beijing: China Machinery Press, 2010.

[12] Gu Xue-song. Financial evaluation model of listed companies by entropy weight based TOPSIS method and solid evidences in petrochemical industry. Value Engineering, 2009(8):160-165.

[13]Luo Jian-qiang, Han Yu-qi, Zhang Yin-ping. Positioning study on customer order decoupling point by entropy weight based TOPSIS method. Industrial engineering and management, 2008(5):83-87. 